\title{
Quantenmechanische Statistik und Korrelationsrechnung
}

\begin{abstract}
Von Fritz Bopp
Aus dem Max-Planck-Institut für Physik der Kaiser-Wilhelm-Gesellschaft in Hechingen (Z. Naturforschg. 2a, 202-216 [1947]; eingegangen am 8. November 1946) ${ }^{1}$

Im 1. Abschnitt wird gezeigt, daß die Annahme einer relativen Häufigkeit über der $p q$-Ebene mit der $\mathrm{H}$ e i s e n b e r g schen Unschärferelation nicht verträglich ist, so daß auf die quantenmechanische Statistik gewöhnliche statistische Methoden nicht anwendbar sind. Abschnitt 2 gibt eine Darstellung der höheren Korrelationsrechnung (nach Gebelein) zur Berechnung einer die Korrelation beschreibenden Matrix. Die reziproke Frage, aus dieser Matrix die Statistik abzuleiten, führt zu einer Verallgemeinerung der gewöhnlichen statistischen Methoden. Abschnitt 3 enthält den Nachweis, daß die quantenmechanische Statistik, ähnlich wie die klassische, einen Sonderfall der verallgemeinerten Statistik darstellt. In Abschnitt 4 wird untersucht, wie sich die Statistik klassischer und quantenmechanischer Gibbs scher Gesamtheiten in die verallgemeinerte Statistik einordnet.
\end{abstract}

1. Unverträglichkeit der Heisenbergschen Unschärferelation mit den gewöhnlichen Methodén der Statistik

$\mathrm{D}$ ie Heisenbergsche Unschärferelation stellt eine statistische Aussage dar über ein durch zwei Merkmale mit kontinuierlich variabler Mannigfaltigkeit von Einstellungsmöglichkeiten $p, q$ gekennzeichnetes Ereignis ${ }^{2}$. Sie besagt, daß für die mittleren Schwankungsquadrate die Ungleichung

$$
\overline{(p-\bar{p})^{2}} \cdot \overline{(q-\bar{q})^{2}} \geq \hbar^{2}
$$

gelte und daß diese erhalten bleibe bei beliebiger kanonischer Transformation der Variabeln $p, q$, speziell also bei der infinitesimalen Transformation

$$
\delta p=-\frac{\partial K}{\partial q}, \quad \delta q=+\frac{\partial K}{\partial p} .
$$

Das Transformationspotential ist dabei willkürlich wählbar.

Da wir zunächst nur den mathematischen Inhalt dieser Aussage studieren wollen, soll einstweilen von allen physikalischen Zusammenhängen abgesehen werden. Wir denken also nicht an Welle und Korpuskel, sondern an einen Punkt in der $p q$-Ebene, über dessen Lage eine statistische Aussage gemacht ist, die genau dieselben Begriffe

1 Abschnitt 4 wurde später (Februar 1947) nachgetragen.

2 Speziell in der Physik spricht man statt von Merkmalen und Möglichkeiten der Einstellung lieber von physikalischen Größen und den Werten, die diese annehmen können. Für die allgemeine Diskussion ziehen wir die statistischen Begriffe vor. benutzt wie geläufige statistische Aussagen über ein durch zwei Merkmale charakterisiertes Ereignis.

Um Ungl. (1) explizit zu formulieren, würde man gewöhnlich die Existenz einer Wahrscheinlichkeitsfunktion $W(p, q)$ voraussetzen.

$$
W(p, q) d p d q
$$

wäre also die Wahrscheinlichkeit dafür, daß sich unser Punkt an der Stelle $p, q$ im Bereich $d p \cdot d q$ befände. Daraus berechneten sich die Mittelwerte und Momente in bekannter Weise. Bei gemäß $\int W(p, q) d p d q=1$ normierter Verteilung wäre

$$
\begin{aligned}
& \bar{p}=\int p W d p d q, \bar{q}=\int q W d p d q \\
& \overline{p^{2}}=\int p^{2} W d p d q, \overline{q^{2}}=\int q^{2} W d p d q ;
\end{aligned}
$$

woraus die in Ungl. (1) eingehenden mittleren Schwankungsquadrate wie gewöhnlich folgen würden. Wir werden jedoch zeigen, daß es keine Verteilungsfunktion $W(p, q)$ gibt, die die Unschärferelation unabhängig vom Bezugssystem richtig wiedergeben kann. Es wird bewiesen, daß man die untere Schranke der Ungleichung bei Annahme einer solchen Verteilungsfunktion durch passende kanonische Transformationen immer weiter herabsetzen kann, was der Voraussetzung widerspricht. Hierin äußert sich die Sonderstellung der quantenmechanischen Statistik ${ }^{3}$.

3 Den obigen Satz hat E. Fues vor Jahren einmal im Anschluß an eine Breslauer Diskussion bewiesen. Seine Beweismethode ist mir heute nicht mehr bekannt, dürfte aber der im Text angegebenen ähnlich gewesen sein. Die hier angestrebten Folgerungen aus dem Satz gehen über die damalige Diskussion hinaus. 
Da die Schwierigkeit jedoch erst entsteht, wenn man das Heisenberg sche Problem mit dem herkömmlichen Kalkül für Ereignisse mit zwei Merkmalen, behandelt, muß man schließen, daß die Methoden dieses Kalküls enger sind als die bei seiner Formulierung benutzten Begriffe. Man darf erwarten, daß man durch Veraligemeinerung zu umfassenderen Methoden kommen kann, und wir wollen versuchen, eine die quantenmechanische
Statistik einschließende Erweiterung zu finden.

Zunächst wollen wir den eben formulierten Satz beweisen. Seien $P=p+\delta p, Q=q+\delta q$ die gemäß Gl. (2) transformierten Variabeln, so erhält man die neuen Mittelwerte analog zu Gl. (4) aus

$$
\bar{P}=\int P(p, q) W(p, q) d p \cdot d q \quad \text { usw. }
$$

Die infinitesimalen Variationen der Mittelwerte lauten also

$$
\begin{aligned}
& \delta \bar{p}=-\int \frac{\partial K}{\partial q} W d p d q, \delta \bar{q}=+\int \frac{\partial K}{\partial p} W d p d q, \\
& \delta \overline{p^{2}}=-2 \int p \frac{\partial K}{\partial q} W d p d q, \delta \overline{q^{2}}=+2 \int q \frac{\partial K}{\partial p} W d p d q .
\end{aligned}
$$

Daraus folgt als Änderung der linken Seite der Unschärferelation der Ausdruck

$$
\delta\left[\left(\overline{p^{2}}-\bar{p}^{2}\right) \cdot\left(\overline{q^{2}}-\bar{q}^{2}\right)\right]=2\left(\overline{p^{2}}-\bar{p}^{2}\right) \int(q-\bar{q}) \frac{\partial K}{\partial p} W d p d q-2\left(\overline{q^{2}}-\bar{q}^{2}\right) \int(p-\bar{p}) \frac{\partial K}{\partial q} W d p d q .
$$

Für eine Verteilungsfunktion, die gerade die untere Grenze der Unschärferelation realisiert, dürfte diese Variation nicht negativ sein, da sonst nach der Transformation die Unschärfe herabgesetzt wäre. Das ist aber nicht der Fall. Setzen wir z. B.

$$
K=\frac{1}{2} \gamma\left(p^{2}-q^{2}\right),
$$

so ergibt sich aus Gl. (5) der Ausdruck

$$
\begin{aligned}
& \delta\left[\left(\overline{p^{2}}-\bar{p}^{2}\right) \cdot\left(\overline{q^{2}}-\bar{q}^{2}\right)\right]= \\
& \quad 2 \gamma\left[\left(\overline{p^{2}}-\bar{p}^{2}\right)+\left(\overline{q^{2}}-\bar{q}^{2}\right)\right](\overline{p q}-\bar{p} \cdot \bar{q}),
\end{aligned}
$$

der negativ wird, wenn wir für $\gamma$ das dem Mittelwert $\overline{p q}-\bar{p} \cdot \bar{q}$ entgegengesetzte Vorzeichen wählen.

Der Beweis ist noch in zwei Punkten zu vervollständigen. Erstens könnten alle für die Unschärferelation zulässigen Verteilungsfunktionen gerade durch die Bedingung $\overline{p q}-\bar{p} \cdot \bar{q}=0$ gekennzeichnet sein. Zweitens brauchte unter diesen Funktionen keine zu sein, die für das Schwankungsprodukt gerade die untere Grenze liefert. Im ersten Falle helfen Veränderungen des Ansatzes in Gl. (6). So folgt z. B. aus $K=\gamma \cdot p^{3}$ :

$$
\begin{aligned}
& \delta\left[\left(\overline{p^{2}}-\bar{p}^{2}\right) \cdot\left(\overline{q^{2}}-\bar{q}^{2}\right)\right]= \\
& \quad 6 \gamma\left(\overline{p^{2}}-\bar{p}^{2}\right)\left(\overline{q p^{2}}-\bar{q} \cdot \overline{p^{2}}\right) \ldots .
\end{aligned}
$$

Da im allgemeinen nicht alle Differenzen solcher Mittelwerte verschwinden können, bleibt unser obiger Schluß bestehen, außer für spezielle Funktionen von der Form $\omega=\omega\left(\frac{p^{2}}{p_{0}{ }^{2}}+\frac{q^{2}}{q_{0}{ }^{2}}\right)$, die gegen kanonische Transformationen nicht invariant sind, so daß die bestmögliche Beobachtung in einem Koordinatensystem in einem andern nicht mehr optimal ist. Was den zweiten Einwand angeht, so muß es jedenfalls Funktionen geben, die beliebig nahe an die untere Grenze heranführen, weil man diese sonst heraufsetzen könnte. Bei Annäherung an diese Grenze streben im allgemeinen die Faktoren von $\gamma$ in den Gln. (6) und (8) - und wenn nicht in diesen, dann wenigstens in ähnlich wie oben passend veränderten Ausdrücken - einem von Null verschiedenen Wert zu, so daß schließlich der Abstand des Schwankungsproduktes von seiner unteren Grenze durch die Transformationsverminderung überkompensiert wird.

Von den physikalischen Betrachtungen über die Unschärferelation herkommend ${ }^{4}$, kann dieses Ergebnis kaum überraschen. Die Unmöglichkeit, $p$ und $q$ gleichzeitig zu messen, weil die Messung der einen Größe die der andern in unkontrollierbarer Weise modifiziert, verhindert offenbar nicht nur eine direkte Bestimmung der Häufigkeitsverteilung in der Phasenebene. Sie macht es auch unmöglich, den Begriff der relativen Häufigkeit auf irgendeine Weise aus den zugänglichen Beobach-

4 W. H e is e n berg, „Physikalische Prinzipien der Quantenmechanik“15, II. u. III. 
tungen indirekt abzuleiten. Daß damit die Unschärferelation nicht ihren Charakter verliert, eine rein statistische Aussage zu sein über die Lage eines Punktes in bezug auf zwei Achsen $p$ und $q$, bleibt in physikalischen Betrachtungen leicht unbeachtet, weil man dort, wo die gewöhnliche Statistik nicht mehr funktioniert, zum Wellenbild übergeht.

Wir wollen die Verhältnisse noch einmal, und zwar an einem finiten, also besser zu übersehenden Beispiel, studieren. Gegenstand der Untersuchung seien Ereignisse, die durch zwei Merkmale $A$ und $B$ mit je zwei Einstellungsmöglichkeiten $a= \pm 1, b= \pm 1$ beschrieben werden. Wie in dem durch die $\mathrm{He}$ is e n berg sche Unschärferelation gegebenen Fall mögen nur Aussagen über Einzelwahrscheinlichkeiten vorausgesetzt werden. Es seien nicht nur die Streuungen, sondern die Gesamtverteilungen gegeben gemäß:

$u(a)=\left\{\begin{array}{l}\cos ^{2} \alpha, \\ \sin ^{2} \alpha,\end{array} \quad v(b)=\left\{\begin{array}{l}\cos ^{2} \beta, \\ \sin ^{2} \beta,\end{array} \quad w(c)=\left\{\begin{array}{l}\cos ^{2} \gamma, \\ \sin ^{2} \gamma .\end{array}\right.\right.\right.$

Darin bedeuten $u(a)$ und $v(b)$ die relative Häufigkeit, mit der die Eigenschaften $a$ und $b$ auftreten, und $w(c)$ die für das Produkt $c=a b$. Während man die Unschärferelation, außer für die Grundvariabeln $p$ und $q$, für eine ganze Gruppe von Transformationen (nämlich für alle kanonisch konjugierten Systeme $P(p, q), Q(p, q)$ formulieren muß, macht bei unserem finiten Beispiel die Angabe der Wahrscheinlichkeitsverteilung für eine einzige Funktion (z. B. für $c=a b$ ) das statistische Problem so bestimmt als möglich.

Es fragt sich, ob man aus den statistischen Angaben in Gl. (9) eine Wahrscheinlichkeitsverteilung

$$
W(a, b)=\left(\begin{array}{ll}
P & Q \\
R & S
\end{array}\right)
$$

ableiten kann. Unter der Annahme der Existenz einer solchen Funktion folgen für die Matrixelemente die Gleichungen

$$
\begin{array}{ll}
P+Q=\cos ^{2} \alpha, & R+S=\sin ^{2} \alpha, \\
P+R=\cos ^{2} \beta, & Q+S=\sin ^{2} \beta, \\
P+S=\cos ^{2} \gamma, & Q+R=\sin ^{2} \gamma .
\end{array}
$$

Ihre Auflösung ergibt

$$
\begin{aligned}
& P=+1 / 2\left(\cos ^{2} \alpha+\cos ^{2} \beta+\cos ^{2} \gamma-1\right), \\
& Q=+1 / 2\left(\cos ^{2} \alpha-\cos ^{2} \beta-\cos ^{2} \gamma+1\right), \\
& R=-1 / 2\left(\cos ^{2} \alpha-\cos ^{2} \beta+\cos ^{2} \gamma-1\right), \\
& S=-1 / 2\left(\cos ^{2} \alpha+\cos ^{2} \beta-\cos ^{2} \gamma-1\right) .
\end{aligned}
$$

Liefert z. B. eine Beobachtung der Größen $a, b$ und $c$ für alle Einstellungsvarietäten Gleichwahrscheinlichkeit, also $\cos ^{2} \alpha=\cos ^{2} \beta=\cos ^{2} \gamma=1 / 2$, so folgt aus Gl. (11) $P=Q=R=S=1 / 4$. Es existiert eine zweiparametrige Wahrscheinlichkeitsverteilung. Erhält man statt dessen

$$
\cos ^{2} \alpha=\cos ^{2} \beta=\cos ^{2} \gamma=1 / 4,
$$

so folgt für die Matrix $W$

$$
W=1 / 8 \cdot\left(\begin{array}{ll}
-1 & +3 \\
+3 & +3
\end{array}\right) .
$$

Es ergibt sich also in diesem Falle keine Wahrscheinlichkeitsverteilung im eigentlichen Sinne, da relative Häufigkeiten wesentlich positive Zahlen sind.

Der Statistiker, der herkömmliche Probleme vor Augen hat, wird zunächst aus Gl. (13) schließen, daß es die in Gl. (12) angenommene Beobachtung in Wirklichkeit nicht geben kann. Die atomphysikalische Erfahrung zeigt jedoch, daß dieser Schluß nicht allgemein richtig zu sein braucht. Aus Gl. (13) kann kein Widerspruch abgeleitet werden, wenn man die beiden Merkmale $A$ und $B$ prinzipiell nicht gleichzeitig messen kann, wenn die Messung jeder Größe eine vorangegangene Messung der andern als zukunftsbestimmenden (hier als $c$-bestimmenden) Faktor zerstört. Das paradoxe Ergebnis in Gl. (13) besagt; daß es dort, wo die Erfahrung solches liefert, z. B. in der Atomphysik, sinnlos oder nur in einem übertragenen Sinne möglich ist, von zweiparametrigen Wahrscheinlichkeitsverteilungen zu sprechen. Gleichwohl wird man auch unter den verallgemeinerten Bedingungen einen Ausdruck brauchen, der die Korrelation zwischen $u, v$ und $w$ beschreibt.

\section{Verallgemeinerung der Korrelationstatistik}

Zur Vorbereitung dieser zu fordernden Verallgemeinerung wollen wir uns mit den Begriffen der gewöhnlichen Korrelationsstatistik vertraut machen. Wir werden sie gleich in einer für das Folgende zweckmäßigen Form schreiben ${ }^{5}$. $X$ und $Y$ seien die beiden ein Ereignis charakterisierenden Merkmale, die beide $N$ Werte $x_{\mu}$ und $y_{\nu}$

${ }^{5}$ Die klassische Korrelationsrechnung ist im Anschluß an H. Ge bel e in, ,Zahl und Wirklichkeit", Leipzig 1943, formuliert. Vergl. auch H. Ge be l e i n, Z. angew. Math. Mech. 21, 364 [1941]. 
$(\mu, \nu=0,1,2 \ldots N-1)^{6}$ annehmen können. $W_{\mu \nu}$ sei eine (echte) zweiparametrige Wahrscheinlichkeitsfunktion. Sie gibt die Wahrscheinlichkeit dafür an, daß $X$ und $Y$ die Werte $x_{\mu}$ und $y_{\nu}$ haben.

$$
u_{\mu}=\sum_{\nu} W_{\mu \nu}, v_{\nu}=\sum_{\mu} W_{\mu \nu}
$$

sind die Einzelwahrscheinlichkeiten für die Größen $X$ und $Y$, die gemäß

$$
\sum_{\mu, \nu} W_{\mu \nu}=\sum_{\mu} u_{\mu}=\sum_{\nu} v_{v}=1
$$

normiert seien. Der Mittelwert einer Funktion $F\left(x_{\mu}, y_{\nu}\right)$ berechnet sich aus

$$
\bar{F}=\sum_{\mu, \nu} F\left(x_{\mu}, y_{\nu}\right) W_{\mu \nu} .
$$

Das Quadrat des Korrelationskoeffizienten lautet nach elementarer Definition

$$
r^{2}=\frac{\overline{(x-\bar{x})(y-\bar{y})}^{2}}{\overline{(x-\bar{x})^{2}} \cdot \overline{(y-\bar{y})^{2}}} \leq 1
$$

Dieser Koeffizient ist nach der $\mathrm{Sch}$ w a r z schen Ungleichung stets $\ll 1$. In der vorliegenden Form hängt er noch von der zufälligen Merkmalbezifferung ab. Bei Merkmaltransformationen ändert sich im allgemeinen sein Wert.

Gebelein löst sich von dieser Willkür, indem er unter der Gesamtheit der möglichen Merkmaltransformationen diejenige aussucht, die einen maximalen Korrelationskoeffizienten ergibt. Das führt zu folgendem Variationsproblem. Unter den Merkmaltransformationen

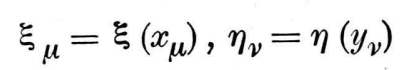

sind diejenigen aufzusuchen, für die der Ausdruck

$$
K^{2}=\frac{\left(\sum_{\mu, \nu} \xi_{\mu} \eta_{\nu} W_{\mu \nu}\right)^{2}}{\sum_{\mu} u_{\mu} \xi_{\mu}^{2} \cdot \sum_{\nu} v_{\nu} \eta_{\nu}^{2}}=\operatorname{Max}
$$

- Die Annahme, daß beide Merkmale $X$ und $Y$ gleichviel Einstellungsmöglichkeiten haben, ist unwesentlich, genügt aber für das Folgende und ist für unsere Zwecke bequem. einen maximalen Wert annimmt. Da die Transformation $\xi_{\mu} \rightarrow \alpha \xi_{\mu}, \eta_{v} \rightarrow \beta \eta_{v}$ den Wert des in $\xi, \eta$ homogenen Ausdrucks 0. Grades $K^{2}$ nicht ändert, können wir uns $\xi_{\mu}$ und $\eta_{\nu}$ o.B.d.A. gemäß

$$
\sum_{\mu} u_{\mu} \xi_{\mu}^{2}=\sum_{\nu} v_{\nu} \eta_{\nu}^{2}=1
$$

normiert denken. Gl. (19) lautet danach einfach

$$
K^{2}=\left(\sum_{\mu, v} \xi_{\mu} \eta_{\nu} W_{\mu, v}\right)^{2}=\operatorname{Max}
$$

oder auch

$$
K=\sum_{\mu, \nu} \xi_{\mu} \eta_{\nu} W_{\mu \nu}=\operatorname{Extr}
$$

da die Wurzel von $K^{2}$ an der Stelle der Maxima mindestens extremale Werte hat. Die Variationen von $\xi_{\mu}$ und $\eta_{\nu}$ ergeben unter Berücksichtigung der Nebenbedingungen in Gl. (20) nach der Lagrangeschen Parametermethode die linearen Gleichungen

$$
\sum_{\nu} W_{\mu \nu} \eta_{\nu}=\varrho u_{\mu} \xi_{\mu}, \sum_{\mu} W_{\mu \nu} \xi_{\mu}=\varrho v_{\nu} \eta_{\nu} \text {. }
$$

Die zu den beiden Nebenbedingungen in Gl. (20) gehörigen Parameter stimmen überein. Denn aus den beiden Gleichungssystemen (22) folgt durch Multiplikoaddition mit $\xi_{\mu}$ und $\eta_{\nu}$ übereinstimmend:

$$
K=\sum_{\mu, \nu} W_{\mu \nu} \xi_{\mu} \eta_{\nu}=\varrho .
$$

Es ergibt sich zugleich, daß der maximale Korrelationskoeffizient ein Eigenwert des Variationsproblems ist.

Auf seine genauere Festlegung kommen wir noch zurück. Zunächst ·ist es zweckmäßig, das Eigenwertproblem in Gl. (22) auf Normalform zu bringen. Setzen wir

$$
\begin{array}{cl}
u_{\mu}=\psi_{\mu}{ }^{2}, v_{\nu}=\chi_{\nu}{ }^{2}, & W_{\mu \nu}=\psi_{\mu} S_{\mu \nu} \chi_{\nu}, \\
\xi_{\mu}=f_{\mu} / \psi_{\mu}, & \eta_{\nu}=g_{\nu} / \chi_{\nu}
\end{array}
$$

so folgt für die Normierung

$$
\sum_{\mu} f_{\mu}{ }^{2}=\sum_{\nu} g_{\nu}{ }^{2}=1,
$$

und Gl. (22) lautet gleich matrizenmäßig ge- 
schrieben:

$$
S g=\varrho f, \quad S^{\prime} f=\varrho g .
$$

$S$ bedeutet darin die Matrix der $S_{\mu \nu}$. $\mathrm{S}^{\prime}$ ist die zugehörige transponierte Matrix; $f$ und $g$ sind die als einspaltige Matrizen geschriebenen Merkmalbezifferungen $f_{\mu}$ und $g_{\nu}$. Iteration führt $\mathrm{zu}$ dem die Korrelation bestimmenden Eigenwertproblem

$$
S S^{\prime} f=\varrho^{2} f, \quad S^{\prime} S g=\varrho^{2} g .
$$

Die Matrix $S$ bezeichnen wir als Korrelationsmatrix. Sie gibt die Verknüpfung der Merkmalbezifferungen $f$ und $g$ einerseits und der Einzelwahrscheinlichkeiten $u$ und $v$ andrerseits.

Letzteres folgt aus Gl. (24). Denn mit Rücksicht auf Gl. (15) liefert Summation über $\mu$ und $\nu$ :

$$
\psi=S \chi, \quad \chi=S^{\prime} \psi .
$$

Die Amplituden $\psi$ und $\chi$ der Einzelwahrscheinlichkeiten $u$ und $v$ - wir werden hier zwangsläufig auf diesen von der Quantenmechanik geprägten Begriff geführt - sind Lösungen des Eigenwertproblems in Gl. (26) zum Eigenwert $\varrho=1$. Sie sagen offenbar nichts über die Korrelation aus und müssen darum bei der Definition des Begriffs des Korrelationskoeffizienten unberücksichtigt bleiben. Erst der nächstgrößte Eigenwert ist nach $\mathrm{G}$ e bele in ein $\mathrm{Maß}$ für die Korrelation und bestimmt den Korrelationskoeffizienten. Wenn er verschwindet, besteht keine Korrelation zwischen den beiden Ereignissen. Sie sind in diesem Falle statistisch unabhängig. Wenn $K=1$ ist, wenn also neben der stets vorhandenen noch eine weitere Lösung zum Eigenwert 1 existiert, herrscht maximale Korrelation. Die Merkmale sind dann einander eindeutig zugeordnet. Nur für den maximalen Korrelationskoeffizienten und im allgemeinen nicht für den elementar definierten in Gl. (17) gelten die eben genannten Sätze vollständig. Beim elementaren Ausdruck sind nur weniger umfassende Aussagen richtig.

Zur Sicherstellung der eindeutigen Zuordnung der Merkmalè genügt, daß ein weiterer Eigenwert des Variationsproblems mit dem trivialen zusammenfällt. Dies folgt aus dem wesentlich positiven Charakter der Wahrscheinlichkeiten $W_{\mu \nu}$. Da ein ähnlicher Satz in der verallgemeinerten Statistik nicht gelten wird, sei hier der Gebele in sche Beweis angeführt ${ }^{5}$ (S. 381). Aus $K=1$ folgt nach Gl. (23)

$$
\begin{aligned}
K & =\sum_{\mu, \nu} W_{\mu \nu} \xi_{\mu} \eta_{\nu} \\
& =\frac{1}{2} \sum_{\mu, \nu} W_{\mu \nu}\left\{\xi_{\mu}{ }^{2}+\eta_{\nu}{ }^{2}-\left(\xi_{\mu}-\eta_{\nu}\right)^{2}\right\}=1
\end{aligned}
$$

oder, wegen der Normierung,

$$
\sum_{\mu, \nu} W_{\mu \nu}\left(\xi_{\mu}-\eta_{\nu}\right)^{2}=0 .
$$

Da in jedem Summanden kein Faktor kleiner als Null sein kann, muß jeder für sich allein verschwinden. Entweder ist $W_{\mu \nu}=0$ oder $\xi_{\mu}=\eta_{\nu}$. Wenn eine ganze Zeile oder Spalte der Matrix $W$ verschwindet, kommt die zugehörige Größe $\xi_{\mu}$ und $\eta_{v}$ gar nicht in der Gleichung vor. Sind mehrere nichtverschwindende Elemente vorhanden, so folgt(wie z.B. aus $W_{\mu \nu}, W_{\mu v^{\prime}} \neq 0: \xi_{\mu}=\eta_{\nu}=\eta_{\nu^{\prime}}$ ), daß verschieden numerierte Werte eines Merkmals einem einzigen Wert des andern Merkmals zugeordnet sind. Danach werden also die Merkmalnummern $\xi_{\mu}$ und $\eta_{\nu}$ in bestimmter, wenn auch im allgemeinen nicht eindeutiger Weise einander zugeordnet. Auf diesem Satz beruht die Möglichkeit, die Korrelation durch eine einzige Zahl (und nicht etwa erst durch die Gesamtheit der Eigenwerte) ungefähr zu kennzeichnen.

Aus der Zusammensetzung von Wahrscheinlichkeiter folgt noch ein bemerkenswerter Satz über Korrelationsmatrizen. Seien $W_{1}(\lambda, \mu)$ und $W_{2}(\mu, \nu)$ die Wahrscheinlichkeiten dafür, daß die Merkmalpaare $X, Y$ und $Y, Z$ bzw. die Werte $x_{\lambda}$, $y_{\mu}$ und $y_{\mu}, z_{\nu}$ annehmen, so lauten die Einzelwahrscheinlichkeiten für jedes Merkmal:

$$
\begin{aligned}
& u\left(x_{\lambda}\right)=\sum_{\mu} W_{1}(\lambda, \mu), \quad v\left(y_{\mu}\right)=\sum_{\lambda} W_{1}(\lambda, \mu) \\
& v\left(y_{\mu}\right)=\sum_{\nu} W_{2}(\mu, \nu), \quad w\left(z_{\nu}\right)=\sum_{\mu} W_{2}(\mu, \nu) .
\end{aligned}
$$

Ferner sind

$$
\frac{W_{1}(\lambda, \mu)}{u\left(x_{\lambda}\right)}, \quad \frac{W_{2}(\mu, \nu)}{v\left(y_{\mu}\right)}
$$

die Zuordnungswahrscheinlichkeiten dafür, daß $Y$ im Zustand $y_{\mu}$ zu finden ist, wenn sich $X$ in $x_{\lambda}$ aufhält, und entsprechend $Z$ in $z_{\nu}$, falls $Y$ in $y_{\mu}$ ist. Die zweiparametrige Wahrscheinlichkeit dafür, daß man $X$ in $x_{\lambda}$ und $Z$ in $z_{v}$ findet, berechnet sich daraus $\mathrm{zu}$ 


$$
W(\lambda, \nu)=u\left(x_{\lambda}\right) \cdot \sum_{\mu} \frac{W_{1}(\lambda, \mu)}{u\left(x_{\lambda}\right)} \cdot \frac{W_{2}(\mu, v)}{v\left(y_{\mu}\right)} .
$$

Nennen wir die zu $u, v, w$ gehörigen Wahrscheinlichkeitsamplituden $\psi, \chi$ und $\omega$ und die $\mathrm{zu} W, W_{1}$ und $W_{2}$ gemäß Gl. (24) gehörigen Korrelationsmatrizen $S, S_{1}$ und $S_{2}$, so folgt aus der letzten Gleichung

$$
S(\lambda, \nu)=\sum_{\mu} S_{1}(\lambda, \mu) S_{2}(\mu ; \nu), S=S_{1} S_{2} .
$$

Bei einer Zusammensetzung von Wahrscheinlichkeiten multiplizieren sich die zugehörigen Korrelationsmatrizen. Diese Eigenschaft haben die Korrelationsmatrizen mit den quantenmechanischen Transformationsmatrizen gemeinsam.

Die Korrelationsrechnung geht von der zweiparametrigen Wahrscheinlichkeitsverteilung $W_{\mu \nu}$ aus. Man kann auch umgekehrt die Korrelationsmatrizen an die Spitze stellen, wie man leicht einsieht, wenn man den zurückgelegten Weg rückwärts geht. Formal wird man dabei stets, auch bei beliebigemAnsatz für $S$, zu einer Funktion $W_{\mu \nu}$ kommen. Aber nur wenn $S$ kennzeichnenden Bedingungen unterworfen ist, kann man $W$ als Wahrscheinlichkeit im Sinne relativer Häufigkeit auffassen. Zunächst dürfen die symmetrisierten Matrizen $S S^{\prime}$ und $S^{\prime} S$ nur Eigenwerte zwischen 0 und 1 haben, unter denen die obere Grenze mindestens einmal vorkommen muß. Ordnen wir diese Eigenwerte in absteigender Folge als Diagonalmatrix an, die wir ihres positiven Charakters wegen mit $D^{2}$ bezeichnen, so muß

sein mit

$$
S S^{\prime}=A D^{2} A^{\prime}
$$

$$
D=\left(\begin{array}{lllll}
1 & 0 & 0 & \cdot & \cdot \\
0 & K_{0} & 0 & \cdot & \cdot \\
0 & 0 & K_{1} & \cdot & \cdot \\
\cdot & \cdot & \cdot & \cdot & \cdot \\
. & . & \cdot & \cdot & \cdot
\end{array}\right)
$$

$A$ bedeutet in diesem Ansatz jene Orthogonalmatrix $\left(A A^{\prime}=1\right)$, die die Matrix $S S^{\prime}$ auf Hauptachsen bringt. Für $S$ selbst folgt daraus:

$$
S=A D B^{\prime},
$$

worin $B$ ebenfalls eine Orthogonalmatrix ist. Die Matrix $S^{\prime} S=B D^{2} B^{\prime}$ wird im allgemeinen von $S S^{\prime}$ verschieden sein, hat aber dieselben Eigenwerte. Aus unseren Ansätzen für $S S^{\prime}$ und $S^{\prime} S$ erhält man vermöge Gl. (27) die Wahrscheinlichkeitsamplituden
$\psi=A \varphi, \quad \chi=B \varphi$
mit $\varphi_{\mu}=\delta_{\mu 1}=\left\{\begin{array}{l}1 \text { für } \mu=1 \\ 0, \mu \neq 1\end{array}\right.$.

Die Normierungsbedingung ist von selbst erfüllt. Die Wahrscheinlichkeiten lauten also nach Gl. (24):

$$
W_{\mu \nu}=\left(\sum_{\lambda} D_{\lambda} A_{\mu \lambda} B_{\nu \lambda}\right) \cdot A_{\mu 1} B_{\nu_{1}} .
$$

Für die Einzelwahrscheinlichkeiten erhält man daraus oder auch unmittelbar aus dem Ansatz für die Amplituden:

$$
u_{\mu}=A_{\mu 1}{ }^{2}, \quad v_{\nu}=B_{\nu 1}{ }^{2} .
$$

Damit ist noch nicht sichergestellt, daß die $W_{\mu \nu}$ relative Häufigkeiten darstellen. Sie können noch immer negative Werte annehmen. Das wollen wir am Beispiel der Doppelalternative zeigen. Es sei

$$
\begin{aligned}
& D=\left(\begin{array}{ll}
1 & 0 \\
0 & K
\end{array}\right)
\end{aligned}
$$

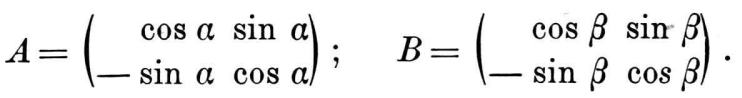

Daraus folgt

$$
\begin{aligned}
W= & \left(\begin{array}{l}
\cos ^{2} \alpha \cos ^{2} \beta, \cos ^{2} \alpha \sin ^{2} \beta \\
\sin ^{2} \alpha \cos ^{2} \beta, \sin ^{2} \alpha \sin ^{2} \beta
\end{array}\right) \\
& +K \sin \alpha \cdot \cos \alpha \cdot \sin \beta \cdot \cos \beta\left(\begin{array}{rr}
1-1 \\
-1 & 1
\end{array}\right) .
\end{aligned}
$$

Wenn $\alpha, \beta$ im Intervall $(0, \pi / 2)$ liegen, können nur die nichtdiagonalen Matrixelemente negativ werden. Das wird durch die Bedingungen

$$
\operatorname{tg} \beta \geq K \cdot \operatorname{tg} \alpha, \quad \operatorname{tg} \alpha \geq K \cdot \operatorname{tg} \beta
$$

verhindert, also wenn $K=1$ ist und wenn der Punkt $\operatorname{tg} \alpha, \operatorname{tg} \beta$ in einem Diagramm mit diesen Koordinaten zwischen den Geraden mit den Steigungsmassen $1 / K \geq K$ liegt. Im Grenzfall $K=1$ erhält man also positives $W$ nur für $\alpha=\beta$. Daraus folgt:

$$
W=\left(\begin{array}{cc}
\cos ^{2} \alpha & 0 \\
0 & \sin ^{2} \alpha
\end{array}\right)
$$

woraus sich die eindeutige Verknüpfung der korrelaten Merkmale ergibt. Ist $\alpha \neq \beta$, so haben wir im Grenzfall $K=1$ :

$$
\begin{aligned}
W=\left(\begin{array}{rrr}
+\cos \alpha & \cos \beta & \cos (\alpha-\beta), \\
+\sin \alpha & \cos \beta & \sin (\alpha-\beta) \\
& -\cos \alpha \sin \beta & \sin (\alpha-\beta) \\
& +\sin \alpha \sin \beta & \cos (\alpha-\beta)
\end{array}\right),
\end{aligned}
$$

also eine Matrix mit einem negativen Element. 
Ohne auf die allgemeine Formulierung der Bedingungen für positive $W_{\mu \nu}$ einzugehen, können wir nun die oben angeschnittene Frage beantworten: Gibt es eine Erweiterung der gewöhnlichen Korrelationsstatistik, die die Statistik der Quantenmechanik mitumfaßt? Die Antwort folgt aus der Umkehrung der Grundfrage der Korrelationsstatistik. Innerhalb der gewöhnlichen Statistik ist die reziproke Frage nicht allgemein lösbar. Wir wollen den Operationsbereich der Statistik so erweitern, daß alle einschränkenden Bedingungen wegfallen, definieren ihn also durch die Korrelationsmatrix, die wir ganz allgemein ansetzen, für die wir sogar komplexwertige Matrixelemente zulassen werden. Die damit auf einem der Mathematik geläufigen Wege gewonnene Verallgemeinerung ist nicht inhaltsleer. Denn sie umfaßt neben der gewöhnlichen Statistik auch die der Quantenmechanik als Spezialfall. Sie liefert außerhalb der gewöhnlichen Statistik statt der zweiparametrigen Häufigkeitsverteilungen entsprechende Funktionen mit negativen Werten, die wir wie jene als statistische Gewichte zur Berechnung von Mittelwerten benutzen. Wenn negative Gewichte vorkommen, sind jedoch die zweiparametrigen Gewichtsfunktionen nicht mehr als relative Häufigkeiten beobachtbar. Die Methoden der verallgemeinerten Statistik gewinnen darum nur dann Bedeutung, wenn die Bestimmung eines Merkmals das statistische System so verändert, daß jede weitere Bestimmung zunächst nur eine Aussage über das veränderte System liefert und keine oder nur eine unvollständige über das ursprüngliche, welches Gegenstand der ersten Untersuchung war.

Den Ausgangspunkt der verallgemeinerten Statistik bildet also die komplexwertige Korrelationsmatrix $S$. Ihre Hermite schen Produkte $S S^{\dagger}$ und $S^{\dagger} S$ haben positiv reelle Eigenwerte $\varrho^{2}$. Mittels der normiert zu denkenden Eigenlösungen $\psi, \chi$ zum Eigenwert $\varrho$ der Gleichungen

$$
\begin{array}{cl}
S^{\dagger} \psi=\varrho \chi, & S \chi=\varrho \psi \\
\text { und } S S^{\dagger} \psi=\varrho^{2} \psi, & S^{\dagger} S \chi=\varrho^{2} \chi
\end{array}
$$

wird die zweiparametrige Gewichtsfunktion der erweiterten Theorie durch die Gleichung

$$
W_{\mu \nu}=\frac{1}{2 \varrho}\left\{\psi_{\mu}^{*} S_{\mu \nu} \chi_{\nu}+\psi_{\mu} S_{\mu \nu}^{*} \chi_{\nu}^{*}\right\}
$$

definiert. Aus ihr folgen in üblicher Weise die wesentlich positiven Einzelwahrscheinlichkeiten

$$
u_{\mu}=\psi_{\mu}^{*} \psi_{\mu}, \quad v_{\nu}=\chi_{\nu}^{*} \chi_{\nu}
$$

und die zu beliebigen reellen Funktionen gehörigen Mittelwerte

$$
\bar{F}=\sum_{\mu, v} F\left(\xi_{\mu}, \eta_{\nu}\right) W_{\mu \nu} .
$$

Gl. (32) ergibt sich nicht aus den vorhergehenden durch Deduktion. Sie enthält eine Definition, die im klassischen Grenzfall offenbar richtig ist, deren Brauchbarkeit in der Quantenstatistik sich aber erst noch erweisen muß.

Dieser Beweis wird durch die Darstellung des Mittelwertes in der Form eines Operator-Erwartungswertes vorbereitet. Setzen wir in Gl. (32) den Ausdruck für $W_{\mu \nu}$ aus Gl. (30) ein und eliminieren wir $\chi$ mittels Gl. (29), so folgt

$$
\bar{F}=\sum_{\mu, v} \psi_{\mu}^{*} \underline{F}_{\mu \nu} \psi_{\nu}=\psi^{\dagger} \underline{F} \psi .
$$

Darin bedeutet $F$ eine $\mathrm{He}$ r mite sche Matrix mit den Komponenten

$$
\underline{F}_{\mu \nu}=\frac{1}{2} \sum_{\lambda}\left(F\left(\xi_{\mu}, \eta_{\lambda}\right)+F^{\prime}\left(\xi_{\nu}, \eta_{\lambda}\right)\right) S_{\mu \lambda} S_{\nu \lambda}^{*} .
$$

Es gibt also in der allgemeinen Korrelationsstatistik und damit bereits in dem Sonderfall der klassischen eine Operatordarstellung für die Mittelwerte.

Die auf 1 normierten Eigenfunktionen $U_{\mu \nu}$ der Matrix $F$, die bezw. zu den Eigenwerten $f_{\nu}$ gehören mögen, bilden gemäß den Gleichungen

$$
\sum_{\underline{F}_{\lambda \mu}} U_{\mu \nu}=U_{\lambda \nu} f_{\nu}, \quad \underline{F} U=U f
$$

eine unitäre Matrix. Definieren wir hierdurch transformierte Wahrscheinlichkeitsamplituden ${ }^{7}$

$$
\omega=U^{\dagger} \psi, \quad \psi=U \omega,
$$

so folgt aus Gl. (33)

d. h.

$$
\bar{F}=\omega^{\dagger} U^{\dagger} \underline{F} U \omega=\omega^{\dagger} f \omega
$$

$$
\bar{F}=\sum_{\lambda} f_{\lambda} \omega_{\lambda}^{*} \omega_{\lambda}
$$

$7 U$ ist hier einerseits die zwischen den Wahrscheinlichkeitsamplituden $\psi$ und $\omega$ vermittelnde Korrelationsmatrix, andererseits eine Transformationsmatrix im quantenmechanischen Sinne. 
In der erweiterten Statistik ist die gewöhnliche Statistik sicher enthalten. Wenn $S_{\mu \nu}$ eine reelle Korrelationsmatrix ist, gehen die Gln. (29) bis (33) in die früher angeschriebenen über, deren Zusammenhang mit den klassischen Formeln wir bereits kennen. Ein einfaches Beispiel aus dem klassischen Bereich mag die wechselseitigen Beziehungen zwischen den Gln. (29) bis (37) deutlich machen. Es sei

$$
S=\left(\begin{array}{ll}
0,48 & 0,36 \\
0,64 & 0,48
\end{array}\right) \text {. }
$$

Daraus folgt mit Rücksicht auf die Gln. (29)

$$
S S^{\prime}=\left(\begin{array}{ll}
0,36 & 0,48 \\
0,48 & 0,64
\end{array}\right), \quad \psi=\left(\begin{array}{l}
0,6 \\
0,8
\end{array}\right), \quad \chi=\left(\begin{array}{l}
0,8 \\
0,6
\end{array}\right) \text {. }
$$

Die Säkulargleichung der Matrix $S S^{\prime}$ lautet $\lambda(\lambda-1)=0$, so daß der zweite Eigenwert verschwindet. Wir haben also ein Beispiel zweier statistisch unabhängiger Einzelwahrscheinlichkeiten. Gemäß Gl. (31) ist

$$
\left(u_{\mu}\right)=\left(\begin{array}{l}
0,36 \\
0,64
\end{array}\right), \quad\left(v_{v}\right)=\left(\begin{array}{l}
0,64 \\
0,36
\end{array}\right)
$$

und nach Gl. (30) erhält man daraus als zweiparametrige Gewichtsfunktion eine echte Wahrscheinlichkeitsverteilung

$$
W=\left(\begin{array}{ll}
0,2304 & 0,1296 \\
0,4096 & 0,2304
\end{array}\right) .
$$

Um die Gültigkeit der Transformationstheorie auch für dieses Beispiel aus der klassischen Wahrscheinlichkeitsrechnung zu zeigen, bilden wir nach Gl. (33) den Mittelwert folgender einfacher Funktion:

$$
F=\left(\begin{array}{ll}
1 & 0 \\
0 & 1
\end{array}\right)
$$

Er ist einerseits gleich der Spur von $W: \bar{F}=0,4608$. Andererseits erhält man nach Gl. (34) als Operatormatrix

$$
\underline{F}=\left(\begin{array}{ll}
0,2304 & 0,2400 \\
0,2400 & 0,2304
\end{array}\right) \text {. }
$$

Sie liefert die Eigenwerte

$$
f=0,2304 \pm 0,24=\left(\begin{array}{l}
+0,4704 \\
-0,0096
\end{array}\right) .
$$

Die Eigenlösungen bilden eine unitäre, hier also speziell eine orthogonale Matrix:

$$
U=\frac{1}{\sqrt{\overline{2}}}\left(\begin{array}{ll}
+1 & +1 \\
+1 & -1
\end{array}\right) .
$$

Sie ergibt nach Gl. (36) die transformierten Wahrscheinlichkeitsamplituden bezw. Wahrscheinlichkeiten:

$$
\omega=\frac{1}{\sqrt{2}}\left(\begin{array}{r}
1,4 \\
-0,2
\end{array}\right), \quad \omega^{2}=\left(\begin{array}{l}
0,98 \\
0,02
\end{array}\right),
$$

aus denen nach Gl. (37) zusammen mit den Eigenwerten wieder der Mittelwert

folgt.

$$
\bar{F}=\Sigma f_{\lambda} \omega_{\lambda}^{*} \omega_{\lambda}=0,4608
$$

3. Anwendung der verallgemeinerten Statistik auf die Quantenmechanik

Über die Ergebnisse der Untersuchungen im vorigen Abschnitt können wir zusammenfassend folgendes sagen: Die Statistik von Ereignissen mit zwei korrelaten Merkmalreihen wird gewöhnlich durch Angabe einer zweiparametrigen Häufigkeitsverteilung erfaßt. Dies setzt jedoch voraus, daß sich die Beobachtung der beiden Merkmale nicht wechselseitig stört. Es ist aber denkbar, daß es Ereignisse gibt, deren beide Merkmale niemals zugleich zugänglich sind, obwohl sie ebenfalls in Korrelation stehen. Auch in einem solchen Falle könnte eine Häufigkeitsverteilung existieren. Aber das ist nicht von vornherein selbstverständlich. Es ist Sache der Beobachtung, und das Ergebnis kann der Annahme einer Häufigkeitsverteilung widersprechen. Die Quantenmechanik gibt dafür Beispiele.

Die Frage, wie man eine allgemeingültige Statistik mit zwei Merkmalreihen aufbauen kann, läßt sich von der Korrelationsrechnung her beantworten. Wenn man nach einer von zufälliger Merkmalbezifferung unabhängigen Definition des Korrelationskoeffizienten sucht, kommt man zu einem Eigenwertproblem, das durch eine Matrix $S$, die Korrelationsmatrix, gekennzeichnet ist. Diese Matrix beschreibt jedoch im allgemeinen nicht nur die Korrelation, sondern enthält die gesamte zweiparametrige Statistik. Allerdings führt nicht jede Matrix zu einer entsprechenden Häufigkeitsverteilung. Doch erlaubt sie allgemein, sogar wenn wir komplexe Elemente zulassen, eine sinnvolle Berechnung von Mittelwerten. Es liegt darum nahe, sie als Basis der gesuchten Verallgemeinerung anzusehen. 
Das ist nur dann möglich, wenn sie die gewöhnliche Statistik mitumfaßt. Und es ist sinnvoll, wenn sie noch etwas mehr leistet, wenn sie z. B. eine rein statistische Auffassung des Kalküls der Quantenmechanik zuläßt. Daß die verallgemeinerte Statistik die klassische mit einschließt, ist nach ihrer Herleitung selbstverständlich. Letztere stellt sich ein, wenn die aus der Korrelationsmatrix abzuleitende Gewichtsfunktion wesentlich positiv ist. In diesem Fall gibt sie die zweiparametrige Häufigkeitsverteilung der Ereignisse an. Die quantenmechanische Statistik erhält man bei anderer Spezialisierung der Korrelationsmatrix $S$. Sie wird in diesem Falle durch zwei Bestimmungen festgelegt, von denen sich die eine auf beliebige Größen und die andere auf speziell einander zugeordnete Größenpaare bezieht. Wir untersuchen zunächst die

Erste Bedingung: Die Wahrscheinlichkeiten für die Werte jeder einzelnen Größe für sich sind statistisch unbestimmt (Unbestimmtheitsforderung).

Diese Forderung ist keineswegs selbstverständlich. Die S-Matrix eines gewöhnlichen Korrelationsproblems liefert dafür ein extrem entgegengesetztes Beispiel. Denn, wenn nicht zufällig ein streng funktioneller Zusammenhang zwischen den beiden Merkmalen besteht, haben die Matrizen $S S^{\dagger}$ und $S^{\dagger} S$ in diesem Falle nur einen Eigenwert +1 . Es gibt also nach Gl. (29) nur je eine Eigenlösung $\psi$ und $\chi \mathrm{zu}$ diesem Eigenwert, wodurch beide Einzelwahrscheinlichkeiten $u$ und $v$ eindeutig bestimmt sind. Wenn eine der beiden durch die $S$-Matrix nicht bestimmt sein soll, müssen sämtliche Eigenwerte von $S S^{\dagger}$ bzw. $S^{\dagger} S$ übereinstimmen und können, weil nach Gl. (30) ein konstanter Faktor vor der S-Matrix nicht in die Gewichtsfunktion eingeht, willkürlich 1 gesetzt werden, so daß wir die S-Matrix der Quantenmechanik unitär annehmen dürfen. Es ist

$$
S^{\dagger} S=S S^{\dagger}=1 \text {. }
$$

Natürlich kann nur eine der beiden Amplituden frei gewählt werden. Die andere berechnet sich nach Gleichung (29) aus

$$
\chi=S^{\dagger} \psi \text {. }
$$

Hierin drückt sich die Korrelation zwischen den beiden Merkmalen aus.
Unter der Voraussetzung unitärer Korrelationsmatrizen nimmt die Operatordarstellung einer Funktion $F\left(\xi_{\mu}, \eta_{\nu}\right)$ nach Gl. (34) eine besonders einfache Gestalt an. Für $F=\xi_{\mu}$ und $F=\eta_{\nu}$ erhält man bzw.

$$
\begin{aligned}
\xi_{\mu \nu} & =\frac{1}{2}\left(\xi_{\mu}+\xi_{\nu}\right) \delta_{\mu \nu}=\xi_{\mu} \delta_{\mu \nu}, \\
\eta_{\mu \nu} & =\sum_{\lambda} \eta_{\lambda} S_{\mu \lambda} S_{\nu \lambda}^{*} .
\end{aligned}
$$

Und für eine zusammengesetzte Funktion gilt allgemein :

$$
\underline{F}=F(\underline{\xi}, \underline{\eta})
$$

An die Stelle einer Zahlenfunktion tritt die entsprechende Operatorfunktion. Produkte sind dabei stets Hermitesch zu schreiben, und zwar so, daß alle $\xi_{-}$- und alle $\eta$-Faktoren je für sich allein zusammenstehen. Es ist leicht $\mathrm{zu}$ verifizieren, daß z. B.

$$
\begin{gathered}
\underline{\eta}_{v}{ }^{2}=\underline{\eta}^{2}, \quad \underline{\xi_{\mu}} \xi_{v}=\frac{1}{2}(\underline{\xi} \underline{\eta}+\underline{\eta} \underline{\xi}), \\
\underline{\xi_{\mu}} \xi_{v}{ }^{2}=\frac{1}{2}\left(\underline{\xi} \underline{\eta}^{2}+\underline{\eta}^{2} \underline{\xi}\right)
\end{gathered}
$$

ist. Die Faktorreihenfolge ist durch Gl. (30) festgelegt.

Die zweite Bedingung wird eine Aussage über spezielle, sogenannte komplementäre Größenpaare enthalten. Wir müssen diese zunächst definieren und in ihren Eigenschaften kennenlernen.

Definition: Die Korrelation komplementärer Größen ist derart, daß vollständige Kenntnis über eine Größe irgendeine Kenntnis über die andere ausschließt.

Es ist sehr bemerkenswert, daß man zur Festlegung des Begriffs der Komplementarität nicht den vollen Inhalt der Unschärferelation braucht, sondern nur eine Aussage für den Grenzfall vollständiger Bestimmtheit einer der beiden komplementären Größen. Im Anschluß an Gl. (39) besagt die Definition: Wenn wir $\psi$ und $\chi$ als ein Paar komplementärer Amplituden auffassen, so müssen alle Matrixelemente von $S$ den konstanten Betrag $1 / \sqrt{N}$ haben. Wenn z. B.das Merkmal $\xi_{\mu}$ durch die Amplitude

$$
\psi_{\mu}=e^{i \alpha} \delta_{\mu \mu_{0}}
$$


genau bestimmt ist, so folgt aus der nach Gl. (39) $\mathrm{zu}$ berechnenden komplementären Amplitude

$$
\chi_{\nu^{\prime}}=S_{\mu_{0} \nu}^{*} e^{i c t}
$$

nur dann Gleichwahrscheinlichkeit für die Werte $\eta_{\nu}$, wenn

$$
\left|S_{\mu_{0} 1}\right|=\left|S_{\mu_{0} 2}\right|=\left|S_{\mu_{0} 3}\right|=\ldots=1 / \sqrt{N}
$$

ist. Die Größe des Betrags ergibt sich dabei aus der Normierung von $\chi$ und der Anzahl der Summanden. Der Ansatz

$S_{\mu \nu}=\frac{1}{\sqrt{N}} \epsilon^{\prime \prime \nu}, \quad(\mu, \nu=0,1,2 \ldots N-1)$,

in $\operatorname{dem} \epsilon=e^{\frac{2 \pi i}{N}}$ die $N$. Einheitswurzel bedeutet, gibt ein Beispiel für eine Komplementaritätsmatrix, das zugleich die Unitaritätsforderung erfüllt. Wegen $\epsilon^{N}=1$ ist nämlich

$\sum_{\mu} S_{\mu \nu} S_{\mu \nu^{\prime}}^{*}=\frac{1}{N} \sum_{\mu} \epsilon^{\mu\left(\nu-v^{\prime}\right)}=\left\{\begin{array}{lll}1 & \text { für } \nu^{\prime}=\nu, \\ 0 & \text { für } \nu^{\prime} \neq \nu .\end{array}\right.$

Bis auf unwesentliche Änderungsmöglichkeiten gibt Gl. (43) den einzigen Ansatz für eine unitäre Komplementaritätsmatrix. Zwar bleiben bei Multiplikation von links und rechts mit unitären Diagonalmatrizen Unitarität der Matrix und Gleichheit der Beträge ihrer Elemente erhalten. Aber bei Rechtsmultiplikation ändern sich nach Gl. (39) die Phasen von $\chi$ entgegengesetzt, so daß die Gewichtsfunktion von der Transformation nicht berührt wird. Bei Linksmultiplikation kann man die Phasen von $\psi$ passend mittransformieren (sogen. Eichtransformation), so daß ebenfalls die Gewichtsfunktion invariant bleibt.

Auf Grund der komplementären Verknüpfung gemäß Gl. (43) kann man über die Matrizen $\underline{\xi}$ und $\eta$ in Gl. (40) noch genauere Aussagen machen. Wir formulieren sie für die beiden Grenzfälle $n=2$ und $n \rightarrow \infty$. Im ersten Falle haben wir, wenn wir für $\xi$ die Werte \pm 1 annehmen:

$$
\begin{aligned}
& \underline{\xi}=\left(\begin{array}{lr}
1 & 0 \\
0 & -1
\end{array}\right), \\
& \underline{\eta}=\frac{1}{2} \cdot\left(\begin{array}{lr}
1 & 1 \\
1 & -1
\end{array}\right)\left(\begin{array}{rr}
1 & 0 \\
0 & -1
\end{array}\right)\left(\begin{array}{rr}
1 & 1 \\
1 & -1
\end{array}\right)=\left(\begin{array}{ll}
0 & 1 \\
1 & 0
\end{array}\right) .
\end{aligned}
$$

Wir erhalten also die beiden $\mathrm{Paulischen} \mathrm{Ma-}$ trizen

$$
\xi=\sigma_{z}, \quad \underline{\eta}=\sigma_{x},
$$

deren Vertauschungsrelation folgendermaßen lautet:

$$
\xi \underline{\eta}-\underline{\eta}=2 \sigma_{y} .
$$

Für $n=2$ geben also gerade die $\mathrm{Paulischen}$ Matrizen ein Beispiel für komplementäre Größen. Die $\mathrm{He}$ is e $\mathrm{n}$ be $\mathrm{r}$ g schen Vertauschungsrelationen sind danach kein spezifisches Kriterium für die Komplementarität. Die Korrelationsmatrix in Gl. (43) ist umfassender ${ }^{8}$.

Im Grenzfall $n \rightarrow \infty$ leiten wir die Operatoren zweckmäßigerweise aus den Mittelwerten ab. Wir gehen von einem Ansatz aus mit endlichen, aber großen Werten ${ }^{9}$ von $N$. Der Mittelwert einer Funktion lautet explizit

$$
\begin{array}{r}
\bar{F}=\frac{1}{2 N} \sum_{\lambda, \mu, v} \psi_{\mu}^{*}\left[F\left(\xi_{\mu}, \eta_{\lambda}\right)+F\left(\xi_{v}, \eta_{\lambda}\right)\right] \\
e^{\frac{2 \pi i}{N}(\mu-v) \lambda} \psi_{\nu} .
\end{array}
$$

Wenn sich die Funktion von Schritt zu Schritt nur wenig ändert und wenn es $\alpha$ bezw. $\beta$ Schritte pro Einheit $\xi_{\mu}$ und $\eta_{\nu}$ gibt, bleibt, wenn wir

$$
\psi_{\mu}=\sqrt{a} \psi(\xi)
$$

und $N \alpha \beta=2 \pi \hbar$ setzen, die Normierung

$$
\begin{aligned}
\sum_{\mu} \psi_{\mu}^{*} \psi_{\mu} & =\int \sqrt{\alpha} \psi^{*}(\xi) \cdot \sqrt{\alpha} \psi(\xi) \frac{d \xi}{\alpha} \\
& =\int \psi^{*}(\xi) \psi(\xi) d \xi=1
\end{aligned}
$$

erhalten, und der Mittelwert lautet

$$
\begin{array}{r}
\bar{F}=\frac{1}{4 \pi \hbar} \int \psi^{*}(\xi)\left[F(\xi, \eta)+F\left(\xi^{\prime}, \eta\right)\right] \\
e^{\frac{i}{\hbar}\left(\xi-\xi^{\prime}\right) \eta} \psi\left(\xi^{\prime}\right) d \xi d \xi^{\prime} d \eta .
\end{array}
$$

${ }^{8}$ In diesem Zusammenhang ist es vielleicht bemerkenswert, daß $\mathrm{H}$ e is enbergs integrale Quantenmechanik zur Theorie der Elementarteilchen (Z. Naturforschg. 1, 608 [1946]) nur Korrelationsmatrizen enthält als Verknüpfung der Wahrscheinlichkeitsamplituden der Besetzungzahlen vor und nach dem Stoß.

9 Die B loc h schen Gleichungen des Ferromagnetismus geben ein Beispiel für endliche Korrelationsmatrizen großer Stufenzahl, für das der Grenzübergang zu ,Spinwellen“ nur eine Näherung. darstellt. 
Daraus folgen für $F=\xi$ und $F=\eta$ die speziellen Ausdrücke

$$
\begin{aligned}
& \bar{\xi}=\int \psi^{*}(\xi) \xi \psi(\xi), \xi, \\
& \bar{\eta}=\frac{\hbar}{i} \int \psi^{*}(\xi) \frac{\partial \psi(\xi}{c} c^{\prime} \xi,
\end{aligned}
$$

die mit den Mittelwerten der den $\mathrm{H}$ e i s e n ber g schen Vertauschungsrelationen

$$
\underline{\xi} \underline{\eta}-\underline{\eta} \underline{\xi}=\frac{\hbar}{i}
$$

genügenden Schrödingerschen Operatoren

$$
\underline{\xi}=\xi \text { und } \underline{\eta}=\frac{\hbar}{i} \frac{\partial}{\partial \xi}
$$

übereinstimmen. Die Konstante $\hbar$ ist in diesen Gleichungen ein Maß für die Korrelation, $1 / \hbar$ ergibt sich durch Grenzübergang aus der Punktdichte in der $\xi_{\eta}$-Ebene. Über die Zusammensetzung der Operatoren ist zu dem oben Gesagten nichts Neues hinzuzufügen. Den Zusammenhang zwischen den komplementären Amplituden erhält man nach einem ähnlichen Grenzübergang aus Gl. (39). Es ist

$$
\chi(\eta)=\frac{1}{\sqrt{2 \pi \hbar}} \int e^{-\frac{i}{\hbar} \xi \eta} \psi(\xi) d \xi .
$$

Das zweiparametrige statistische Gewicht lautet also

$$
\begin{array}{r}
W(\xi, \eta)=\frac{1}{\sqrt{2 \pi \hbar}}\left[\psi^{*}(\xi) e^{-\frac{i}{\hbar} \xi \eta} \chi(\eta)\right. \\
\left.+\psi(\xi) e^{-i-\frac{\xi}{\hbar} \eta} \chi^{*}(\eta)\right] .
\end{array}
$$

Gemäß Gl. (32) folgen aus ihm die Gln. (47) durch gewöhnliche Mittelbildung ${ }^{10}$.

Die quantenmechanische Statistik wird erst durch die Angabe vervollständigt, welche Größenpaare komplementär sind, und im Falle kontinuierlicher Variabeln durch die Bestimmung der Konstanten $\hbar$. Die zweite Bedingung lautet daher folgendermaßen :

Z weite Bedingung: Die kanonisch konjugierten Variabeln des Phasenraums sind komplementär und $2 \pi \hbar=6,62 \cdot 10^{-2 \gamma} \mathrm{erg} \cdot \mathrm{sec}$ (Komplementari-

$$
\text { tätsprinzip). }
$$

Daraus folgt die mittlere Energie sofort aus der Hamilton-Funktion $H=H(p, q)$ des klassi- schen Problems

$$
\bar{E}=\int H(p, q) W(p, q) d p d q .
$$

Diese ist nach Gl. (33) identisch mit dem quantenmechanischen Operatorausdruck

$$
\bar{E}=\int \psi^{*}(q) H\left(\frac{\hbar}{i} \frac{\partial}{\partial q}, q\right) \psi(q) d q .
$$

Die letzte Bedingung ist nur dann in sich widerspruchsfrei, wenn die Eigenschaft der Komplementarität gegen kanonische Transformationen invariant ist. Dieses Faktum ist aus der Quantenmechanik geläufig. Es ergibt sich etwa aus der Invarianz der Vertauschungsrelationen ${ }^{11}$.

Mit der Formulierung der Statistik sind die Bewegungsgesetze noch nicht vollständig bestimmt. Denn eine der beiden Wahrscheinlichkeitsamplituden bleibt nach Bedingung 1 statistisch unbestimmt. Die Ergänzung ist jedoch für die gewöhnliche Quantenmechanik ohne Problematik. Zunächst kann man noch aus dem statistischen Verhalten eine Aussage über die zeitliche Änderung der Wahrscheinlichkeitsamplituden machen. Seien $\psi$ und $\psi_{0}$ die Amplituden, die der Variabeln $q$ zur Zeit $t$ und $t=0$ zugeordnet sind, so muß die beide Größen verknüpfende Korrelationsmatrix nach der ersten Bedingung unitär sein. Es ist also

$\psi=S_{t} \psi_{0}, \quad \psi_{0}=S_{t}^{+} \psi ; \quad\left(S_{t}^{\dagger} S_{t}=S_{t} S_{t}^{\dagger}=1\right)$.

Für infinitesimale Änderungen folgt daraus die Gleichung

$\dot{\psi}=-i J \psi$ mit $J=i \dot{S}_{t} S_{t}^{\dagger}, \quad\left(J^{\dagger}=J\right)$,

in der $J$ eine $\mathrm{Hermitesche} \mathrm{Matrix} \mathrm{bedeutet.}$

Die Bestimmung der infinitesimalen Korrelationsmatrix $J$ setzt ein neues Prinzip voraus, das wir folgendermaßen formulieren:

Dritte Bedingung: Im Grenzfall verschwindender Korrelation $(\hbar \rightarrow 0)$ geht Gl. $(53)$ in die H a milto $n$ - J a c o b i sche Differentialgleichung der klassischen Mechanik über (Korrespondenzprinzip).

Die Ausrechnung der Näherung ist aus den Elementen der Quantenmechanik geläufig (0.Nä-

${ }^{10}$ Dieser Ausdruck unterscheidet sich von dem Dirac-Neumannschen „Operator“ der Wahrscheinlichkeit (vergl. Abschnitt 4).

$11[P(\underline{p}, q), Q(\underline{p}, q)]=[p-\partial K / \partial \underline{q}, \underline{q}+\partial K / \partial \underline{p}]=[p, q]$ für infinitesimale Transformationen. 
herung des $W B K$-Verfahrens) und ergibt:

$$
\begin{gathered}
J=\frac{1}{\hbar} H(\underline{p}, \underline{q}), \\
-\frac{\hbar}{i} \frac{\partial \psi}{\partial t}=H(\underline{p}, \underline{q}) \psi(q),
\end{gathered}
$$

also die zeitabhängige Schröd ing e r - Gleichung. Eine besonders einfache Gestalt hat das Korrespondenzprinzip im Falle stationärer Lösungen. Es lautet: Die mit dem statistischen Gewicht in Gl. (50) berechnete mittlere Energie aus Gl. (51) ist extremal. Der mit Gleichung (51) identische Ausdruck in Gl. (52) führt danach mit Rücksicht auf die Nebenbedingung $\int S \psi^{*} \psi d q=1$ unmittelbar zur zeitunabhängigen Sch rö ding e r-Gleichung.

4. Einordnung quantenmechanischer Gibbsscher Gesamtheiten ${ }^{12}$ in die verallgemeinerte Statistik

Die Bedeutung der Komplementaritätsmatrix in Gl. (43) ist umfassender, als es bisher zum Ausdruck kommt. Sei $S_{\mu \nu}$ eine beliebige Korrelationsmatrix und seien $\psi_{\mu}$ und $\chi_{\nu}$ die zugehörigen Wahrscheinlichkeitsamplituden, die bei nichtentarteten Eigenwertproblemen gemäß Gl. (29) wohlbestimmt sind, so kann man die Gewichtsfunktion aus Gleichung (30) folgendermaßen

$$
W_{\mu \nu}=\frac{1}{2 \varrho}\left[\psi_{\mu}^{*} S_{\mu \nu} \chi_{\nu}+\psi_{\mu} S_{\mu \nu}^{*} \chi_{\nu}^{*}\right]=\frac{1}{2 \sqrt{N}} \sum_{n} p_{n}\left[\psi_{\mu}^{*}(n) \epsilon^{+\mu \nu} \chi_{\nu}(n)+\psi_{\mu}(n) \epsilon^{-\mu \nu} \chi_{\nu}^{*}(n)\right]
$$

nach zwei komplementären Orthogonalsystemen $\psi(n)$ und $\chi(n)$ entwickeln. $\frac{1}{\sqrt{N}} \epsilon^{\mu \nu}$ bedeutet darin die Komplementaritätsmatrix aus Gl. (43), $\chi(n)$ und $\psi(n)$ sind gemäß Gl. (39) durch

$$
\frac{1}{\sqrt{N}} \epsilon^{\mu \nu}
$$

verknüpft und folgen aus den Gleichungen

$$
\frac{1}{\varrho} \psi_{\mu}^{*} S_{\mu \nu} \chi_{\nu}=\frac{1}{\sqrt{N}} \sum_{n} p_{u} \psi_{\mu}^{*}(n) \epsilon^{\mu \nu} \chi_{\nu}(n)
$$

Multiplizieren wir mit $\epsilon^{\left(\mu^{\prime}-\mu\right) v}$, so ergibt sich bei anschließender Addition über v mit Rücksicht auf

$$
\frac{1}{\sqrt{N}} \sum_{v} \epsilon^{\mu^{\prime} v} \chi_{\nu}(n)=\psi_{\mu^{\prime}}(n)
$$

folgende Beziehung

$$
\begin{aligned}
& \frac{1}{\varrho} \sum_{v} \psi_{\mu}^{*} S_{\mu \nu} \epsilon^{\left(\mu^{\prime}-\mu\right) v} \chi_{\nu} \\
& =\sum_{n^{\prime}} p_{u^{\prime}} \psi_{\mu}^{*}\left(n^{\prime}\right) \psi_{\mu^{\prime}}\left(n^{\prime}\right),
\end{aligned}
$$

aus der nach Multiplikoaddition mit $\psi_{\mu}(n)$ das unser Orthogonalsystem bestimmende Eigenwertproblem folgt. Es ist

$$
\sum_{\mu} P_{\mu^{\prime} \mu} \psi_{\mu}(n)=p_{n} \dot{\psi}_{\mu^{\prime}}(n) .
$$

Darin bedeutet $P$ die Matrix

$$
P_{\mu \mu^{\prime}}=\frac{1}{\varrho} \sum_{\nu} \psi_{\mu^{\prime}}^{*} S_{\mu^{\prime} v} \epsilon^{\left(\mu-\mu^{\prime}\right) v^{\prime}} \chi_{\nu} .
$$

Berechnen wir den Mittelwert einer Funktion $\boldsymbol{F}^{\prime}\left(\xi_{\mu}, \eta_{\nu}\right)$ mittels Gl. (57), so folgt unter Berücksichtigung der Gln. (33), (34) und (41):

$$
\bar{F}=\sum_{n} p_{n} \psi^{+}(n) F(\underline{\xi}, \underline{\eta}) \psi(n),
$$

also ein Ausdruck, der formal mit dem quantenmechanischen Mittelwert einer Gibbsschen Gesamtheit übereinstimmt ${ }^{13}$. Unter Benutzung der Eigenfunktionen können wir die Matrix $P$ auch noch in der Form

$$
P_{\mu \mu^{\prime}}=\sum_{n} p_{u} \psi_{\mu}(n) \psi_{\mu^{\prime}}^{*}(n)
$$

schreiben. Sie führt zu dem für Mittelwerte geläufigen Ausdruck: '

$$
\bar{F}=\sum_{\mu, \mu^{\prime}} \underline{F}_{\mu} \mu^{\prime} P_{\mu^{\prime} \mu}=\operatorname{Spur}(\underline{F} P) .
$$

12 Die folgenden statistischen Betrachtungen sind durch Diskussionsbemerkungen von Hrn. Dr. Pikkert (Math. Inst. Tübingen) und Hrn. Dr. Moli ère angeregt, für die ich beiden herzlich danke.

13 Vergl. hierzu P. A. M. Dirac, Principles of Quantum Mechanics, 2. Aufl.; § 37, Oxford 1935; J. v. Neumann, Mathematische Grundlagen der Quantenmechanik, Kap. IV ff.; Springer 1932; G. Moli èr e u. M. Delbrück, S.-B. preuß. Akad. Wiss., Physik.math. Kl. 1936, Nr. 1. 
Wir können danach formal jede Wahrscheinlichkeitsverteilung - auch eine klassische - auffassen als eine Gibbssche Gesamtheit quantenmechanischer Systeme. Da jedoch die Matrix $P$ in Gl. (59) im allgemeinen nicht Hermitesch sein wird, brauchen die Eigenwerte $p_{n}$ weder positiv noch reell zu sein. Nur wenn letzteres zutrifft, nur wenn $P$ lauter reelle, nicht negative Eigenwerte hat, können wir diese als Wahrscheinlichkeiten, als relative Häufigkeiten für die einzelnen in der Gibbsschen Gesamtheit vorhandenen Quantenzustände auffassen. Das Ergebnis unserer Betrachtung lautet also: Die Gewichtsfunktion für eine Gibbssche Gesamtheit quantenmechanischer Systeme stellt eine umfassendere Realisierung unserer verallgemeinerten Statistik dar als der in Abschnitt 3 untersuchte „,reine Fall“. Aber nicht jeder Ansatz einer Gewichtsfunktion der verallgemeinerten Statistik läßt sich als echte Gibbssche Gesamtheit quantenmechanischer Systeme auffassen. Das ist nur möglich, wenn die Zustandsgewichte $p_{n}$ reelle, nicht negative Zahlen sind und damit als $\mathrm{Zu}$ standswahrscheinlichkeiten aufgefaßt werden können. Die beiden bekannten Realisierungsmöglichkeiten der verallgemeinerten Statistik lassen sich hiernach in drastischer Weise einander gegenüberstellen: Klassische statistische Gesamtheiten sind durch positive Gewichtsfunktionen, quantenmechanische statistische Gesamtheiten durch positive Zustandsgewichte gekennzeichnet. Diese beiden Möglichkeiten stellen jedoch keine Alternative dar. Erstens gibt es Gesamtheiten, die beiden Bedingungen genügen, die sich also zugleich klassisch und quantenmechanisch verstehen lassen. Zweitens kann man Gesamtheiten angeben, die weder klassisch noch quantenstatistisch gedeutet werden können.

Diese verschiedenen Möglichkeiten sollen an den speziellen, am Ende der Abschnitte 1 und 2 diskutierten Doppelalternativen erläutert werden. Die Gewichtsfunktionen

$$
W=\left(\begin{array}{ll}
A & B \\
C & D
\end{array}\right)
$$

(es ist hier zweckmäßig, für die Matrixelemente neue Buchstaben $\mathrm{zu}$ benutzen) sind in beiden Fällen reell, so daß wir auch $S$, $\psi$ und $\chi$ reell annehmen können. In diesem Fall lautet Gl. (59) einfach
Daraus folgt

$$
P_{\mu, \mu^{\prime}}=\sum_{v} W_{\mu^{\prime} v}(-1)^{\left(\mu-\mu^{\prime}\right) v} .
$$

$$
P=\left(\begin{array}{ll}
A+B & C-D \\
A-B & C+D
\end{array}\right),
$$

so daß die Gewichtsfaktoren

$$
p=1 / 2 \pm 1 / 2 \sqrt{1-8(A \nu+B C)}
$$

sind. Die beiden klassischen Beispiele $A=B=C$ $=D=1 / 4$ zwischen den Gln. (11) u. (12) und $A=D=0,2304 ; B=0,1296$ und $C=0,4096$ am Ende von Abschnitt 2 liefern ebenso wie das Beispiel in Gl. (13) lauter positive $p$ (nämlich bezw. $p_{1}=p_{2}={ }^{1 / 2} ; p_{1,2}=0,5 \pm 0,1941$ und $p_{1}={ }^{3 / 4}, p_{2}={ }^{1 / 4}$ ), lassen sich also sämtlich quantenstatistisch verstehen, die beiden ersten Beispiele außerdem - wie wir oben gesehen haben - auch klassisch. Der Ansatz $A=D=3 / 8, B=C=1 / 8$ liefert ein rein klassisches Beispiel, denn die Zustandsgewichte $p=0,5 \pm 0,25 i$ sind komplex.

$$
P=\frac{1}{16}\left(\begin{array}{rr}
7 & -3 \\
5 & 7
\end{array}\right) \quad \text { mit } p=0,5 \pm 0,125 i
$$

ist offenbar weder klassisch noch quantenstatistisch deutbar. Es ergibt sich aus Gl. (11) mit $\cos ^{2} \alpha=1 / 4, \quad \cos ^{2} \beta=3 / 4, \quad \cos ^{2} \gamma=7 / 8$. Unsere verallgemeinerte Statistik reicht also, auch wenn wir Gibbssche Gesamtheiten quantenstatistischer Systeme in Betracht ziehen, weiter als bekannte Ansätze.

Sie führt uns damit zu einigen über die Quantenstatistik hinausweisenden Fragen. Kann man etwa aus dem Ganzen noch andere ausgezeichnete Spezialfälle herausheben? Gibt es Beispiele, die weder von der klassischen noch von der quantenmechanischen Statistik erfaßt werden? Wir stellen diese Frage, ohne sie gegenwärtig beantworten zu können. Es ist nicht einmal leicht anzugeben, welche Art statistischer Beobachtung nach Realisierungen der verallgemeinerten Statistik zu suchen erlaubt. Die Angabe einer Reihe von Einzelwahrscheinlichkeiten reicht allein nicht aus, um eine Aussage über die Korrelationsmatrizen zu gewinnen. Ohne weitere Kenntnisse kann man sie stets als statistisch unabhängig betrachten. Erst wenn man etwas mehr weiß - wie im Beispiel am Ende von Abschnitt 1, in $\operatorname{dem} c$ als Funktion von $a, b$ gegeben ist $(c=a b)-$, erhält man Bestimmungsgleichungen für die Korrela- 
tionsmatrizen. Hier wird man die mathematische Analyse noch weiter führen müssen, ehe man entscheiden kann, ob es neben der gewöhnlichen und der quantenmechanischen noch andere Arten der Korrelationsstatistik gibt, und ehe man es unternehmen kann, nach readen Beispielen für die neue Statistik zu suchen.

Formulieren wir zum Schluß, was aus den obigen Betrachtungen für die Quantenmechanik folgt! ${ }^{14}$ Die quantenmechanische Beschreibung eines atomaren Systems ist eine rein statistische. Sie gibt im Prinzip für jede einzelne Größe, nicht aber für die Gesamtheit aller Größen zugleich, die relative Häufigkeit ihrer Werte. Sämtliche Größen stehen miteinander in Korrelation. Gemäß der allgemeinen Korrelationsstatistik werden die Wahrscheinlichkeitsamplituden der einzelnen Größen durch Korrelationsmatrizen, gewöhnlich Transformationsmatrizen genannt, miteinander verknüpft. Diese Matrizen sind speziell in der Quantenmechanik unitär (1. Bedingung). Letzteres bedeutet physikalisch, daß von allen Einzelwahrscheinlichkeiten eine nicht durch die Statistik, sondern erst durch eigentlich physikalische Bestimmungsstücke (Hamilton-Funktion, Anfangsbedingungen, Randbedingungen $u$. dergl.) festgelegt wird. Hierin unterscheiden sich quantenstatistische Probleme von solchen der gewöhnlichen Korrelationsstatistik. Alle andern Größen folgen aus der einen, die willkürlich ausgewählt werden kann, gemäß ihrer Korrelation.

Unter den möglichen Korrelationen spielt die komplementäre, durch Gl. (43) definierte, eine besondere Rolle. Sie verknüpft die kanonisch konjugierten Variabeln derart (2. Bedingung), daß vollständige Kenntnis einer Größe jede Kenntnis über die andere ausschließt. Im Falle kontinuierlicher Variabeln ist die Komplementarität durch Gl. (49) gegeben, und die durch Gl. (33) definierten Operatoren komplementärer Größen genügen den He is e n be r g schen Vertauschungsrelationen in Gl. (48). Gl. (49) ist von der Form eines Fresnelschen Beugungsintegrals. Es liefert unmittelbar die vollständige Unschärferelation. Von hier aus finden alle Diskussionen von Gedankenexperimenten, die vom Korpuskel- zum Wellenbild übergehen, ihre statistische Rechtfer-

14 Es verdient bemerkt zu werden, daß H. G. K ̈̈ $ß$ ner die quantenmechanische und die Gebele in sche Statistik in einer der vielen anregenden Randbemerkungen in ,Principia Physica“, S. 240, Vandenhoeck \& Ruprecht [1946], zusammen nennt. tigung. Nur kann man schwerlich noch von einer Dualität in dem Sinne sprechen, daß ein Elektron je nach den Versuchsbedingungen bald als Welle, bald als Teilchen erscheine. Wir haben in der hier entwickelten Statistik stets das. Teilchen vor uns, dessen Bewegung jedoch nach Wahrscheinlichkeitsgesetzen erfolgt. Diese werden durch Wellen im Konfigurationsraum beschrieben.

Neben den Korpuskeln gibt es im dreidimensionalen Anschauungsraum auch noch Wellen im klassischen Sinne ${ }^{15}$. Von diesen Wellen einer klassischen Feldtheorie ausgehend, kann man, wie die Quantentheorie der Wellenfelder zeigt, ähnlich wie oben zu einer statistischen Beschreibung übergehen. Man weiß, daß man dabei ebenfalls zu Wellen im Konfigurationsraum kommt und daß letztere nach passender Variabelntransformation mit den Konfigurationswellen einer entsprechenden Partikeltheorie übereinstimmen. Man kann also wenigstens prinzipiell zu jeder klassischen Partikeltheorie eine in der statistischen Beschreibung äquivalente klassische Wellentheorie angeben und umgekehrt, so daß man jeden quantenstatistischen Vorgang rein partikelmäßig oder rein wellenmäßig beschreiben kann. Danach wäre es z.B. bei der Diskussion der Heisenb.erg schen Gedankenexperimente keineswegs notwendig, vom Partikel- zum Wellenbild überzugehen, wenn man nur den statistischen Komplementaritätsbegriff benutzt. Es ist aber möglich und vielleicht zur anschaulichen Beschreibung der Komplementarität bequem.

Jede statistische Aussage enthält subjektive Elemente. Denn sie ist nicht nur von den Objekten her bestimmt, sondern auch von dem Umfang unseres Wissens über diese. Wenn man in quantenmechanischen Erörterungen von einem Einbruch des Subjekts in die objektive Welt der Physik spricht, so meint man damit allerdings etwas anderes. Wegen der Unmöglichkeit der Ver-

15 Die folgenden Überlegungen ergaben sich aus einer Diskussion mit Herrn Prof. H e is e $n$ be r g, für die ich ihm herzlich danken möchte. Man vergl. hierzu H e i s e n'b er g, „Physikalische Prinzipien“, Hirzel [1930], VI 6/8, und v. W e i z s ä c k e r, ,Zum Weltbild der Physik“, Kant-Vortrag, Hirzel [1945], ebenso dessen demnächst erscheinenden Aufsatz: „Möglichkeit und Unendlichkeit“, in dem unabhängig die Quantentheorie als Ausdruck einer neuartigen Statistik erkannt wird. Auch ihm sei für Diskussionen und für die Überlassung des Aufsatzes vor seiner Publikation herzlich gedankt. Dieser Dank gilt auch für einige in dieselbe Richtung weisende briefliche Anmerkungen von Hrn. Geh. Rat $\mathrm{S}$ o m m e r f e l d. 
einigung dualer Seinsweisen im Objekt hängt es vom Beobachter $a b$, in welcher Gestalt ihm das Objekt entgegentritt. Die quantenmechanische Statistik ist also nicht nur wegen unseres beschränkten Wissens subjektgefärbt, sondern auch wegen der Gleichwertigkeit verschiedener $G e$ stalten zur Beschreibung des Seins. Dabei ist zu beachten, daß die Behauptung der Gleichwertigkeit der beiden Gestalten: Korpuskel und Welle, eine objektive Aussage darstellt, die aus der speziellen Art der Korrelation, nämlich der Kom- plementarität, folgt. Die Heis en bergschen Gedankenexperimente beweisen in diesem Zusammenhang, daß man sich der objektiv bestehenden Bindung durch die Komplementarität auf keine Weise entziehen kann. Sie geben uns ebenso von der Komplementarität Kunde wie z. B. die Stabilität der Elektronenbahnen in unberührten Wasserstoffatomen.

Die vorstehenden Untersuchungen sind dureh Diskussionen über v. We i z sä ckers oben zitierte ${ }^{15}$ Vorträge mit Hrn. Prof. Fues angeregt, für die ich auch an dieser Stelle herzlich danken möchte.

\title{
Zur Theorie der Neuen Sterne
}

\author{
Von Gerhard Miczaika \\ Aus der Badischen Landessternwarte Heidelberg-Königstuhl \\ (Z. Naturforschg. 2 a, 216-219 [1947]; eingegangen am 30. September 1946)
}

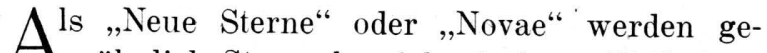
Awöhnlich Sterne bezeichnet, deren Helligkeit sehr plötzlich, etwa in einigen Stunden oder ein bis zwei Tagen, um mehrere Größenklassen ansteigt, um dann erheblich langsamer wieder zum Ausgangswert, meist unter sekundären Schwankungen, zurückzukehren. Aus den spektroskopischen Befunden geht hervor, daß der Ausbruch mit einer gewaltigen Vergrößerung der Sternoberfläche verbunden ist, die im wesentlichen als eine Vergrößerung der Atmosphäre des Sterns aufzufassen sein dürfte. Violettverschiebung der Absorptionslinien, Verbreiterung der Emissionslinien und schließlich Auftreten typischer Nebellinien sind die wichtigsten Charakteristika für das Ablaufen des Novaprozesses. Zur Kennzeichnung einer Nova müssen beide Kriterien, plötzlicher Helligkeitsanstieg und spektrale Merkmale, nachweisbar sein.

Die bei einem Ausbruch abgegebene Energie ist nur ein geringer Bruchteil des potentiellen Energievorrats eines Sterns, der der Größenordnung nach etwa $10^{49} \mathrm{erg}$ beträgt. B i e r m a n $n^{1}$ schätzt die bei einem Ausbruch abgegebene Energie aus der Lichtkurve auf etwa $10^{44} \mathrm{erg}$. Massenmäßig dürfte ebenfalls nur ein kleiner Teil des Sterns an der Aufblähung und Ausbildung einer ausgedehnten Atmosphärenschicht teilnehmen.
\end{abstract}

1 L. B i erm a n n, Z. Astrophysik 18, 344 [1939].
Das eindrucksvolle Phänomen der Novae ist häufig und bereits sehr früh Gegenstand von Hypothesen über seinen Ursprung gewesen, ohne daß bisher eine befriedigende Erklärung gefunden worden wäre. Die zur Zeit zweifellos plausibelsten Vorstellungen haben Uns öld ${ }^{2}$ und später B i e r m a $n n^{1}$ entwickelt.

Uns öld weist darauf hin, daß in einer Sternatmosphäre eine herabsinkende Gasmasse nicht nur ihren Druck und ihre Temperatur, sondern auch ihren Ionisationsgrad ändert. Bei Temperaturerhöhung würde also die Translationsenergie der Teilchen vergrößert und Ionisationsarbeit geleistet. Das Verhalten des Gases nähert sich dem eines mehratomigen, da das effektive Verhältnis der spezifischen Wärmen $\bar{x}<5 / 3$ wird. Unter der Voraussetzung, daß eine Schicht hauptsächlich aus Wasserstoff aufgebaut ist, und Annahme plausibler Werte für Temperatur und Massenabsorptionskoeffizient sinkt $\bar{x}$ unter Umständen sogar unter ${ }^{4} / 3$. Dies bedeutet bekanntlich instabiles Strahlungsgleichgewicht und thermodynamische Stabilität der ausgleichenden Konvektionsströme. Un söld glaubt nun, daß die Instabilität auch anders zum Ausgleich kommen kann. Der thermodynamisch instabile Zustand eines Teils des Sterns bedeutet noch nicht dynamische Instabilität des Ganzen. Eine kleine Störung jedoch

2 A. Unsöld, Z. Astrophysik 1, 138 [1930]. 